\title{
Reflets
}

Revue d'intervention sociale et communautaire

\section{Le rôle des intervenantes du secteur de la violence faite aux femmes dans le développement des services en français en Ontario : une étude exploratoire}

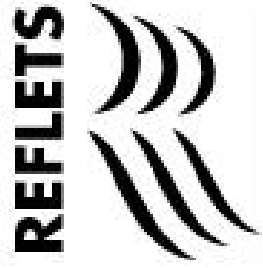

\author{
Linda Cardinal, Marie-Luce Garceau, Ghislaine Sirois et Nathalie Plante
}

Volume 21, numéro 1, printemps 2015

Violences faites aux femmes et contextes minoritaires

URI : https://id.erudit.org/iderudit/1032548ar

DOI : https://doi.org/10.7202/1032548ar

Aller au sommaire du numéro

Éditeur(s)

Reflets, Revue d'intervention sociale et communautaire

ISSN

1203-4576 (imprimé)

1712-8498 (numérique)

Découvrir la revue

Citer cet article

Cardinal, L., Garceau, M.-L., Sirois, G. \& Plante, N. (2015). Le rôle des intervenantes du secteur de la violence faite aux femmes dans le développement des services en français en Ontario : une étude exploratoire. Reflets, 21(1), 40-72. https://doi.org/10.7202/1032548ar
Résumé de l'article

L'article porte sur le rôle des intervenantes francophones du secteur de la violence faite aux femmes (VFF) dans le développement de services en français en Ontario. Depuis les années 1990, ce secteur a fait des avancées importantes dans le développement de services en français, et peut désormais venir en aide à de plus en plus de femmes francophones. Or, il existe peu d'études portant sur le rôle des intervenantes dans le développement de ces services en français. Cette première analyse de la situation repose sur les résultats d'entretiens menés auprès de 14 intervenantes francophones du secteur de la VFF. Ces entretiens permettent de commencer à prendre la mesure de leur rôle dans le développement des services en français dans le secteur de la VFF. Les données portent sur les modes d'action des intervenantes ainsi que les savoirs ou apprentissages qu'elles tirent de leurs efforts afin de les transmettre à d'autres et ainsi encourager la réflexion sur les enjeux liés au développement des services en français en Ontario.
Tous droits réservés (C) Reflets, Revue d’intervention sociale et communautaire, 2015
Ce document est protégé par la loi sur le droit d'auteur. L'utilisation des services d'Érudit (y compris la reproduction) est assujettie à sa politique d'utilisation que vous pouvez consulter en ligne. 


\section{Le rôle des intervenantes du secteur de la violence faite aux femmes dans le développement des services en français en Ontario : une étude exploratoire}

\section{Linda Cardinal}

Professeure titulaire, École d'études politiques, Chaire de recherche sur la francophonie et les politiques publiques, Université d'Ottawa

\section{Marie-Luce Garceau}

Professeure titulaire, École de service social, Université Laurentienne

Ghislaine Sirois

Chercheuse communautaire et analyste, Gatineau

Avec la collaboration de Nathalie Plante

Alliance de recherche Les savoirs de la gouvernance communautaire, Université d'Ottawa

\section{Résumé}

L'article porte sur le rôle des intervenantes francophones du secteur de la violence faite aux femmes (VFF) dans le développement de services en français en Ontario. Depuis les années 1990, ce secteur a fait des avancées importantes dans le développement de services en français, et peut désormais venir en aide à de plus en plus de femmes francophones. Or, il existe peu d'études portant sur le rôle des intervenantes dans le développement de ces services en français. Cette première 
analyse de la situation repose sur les résultats d'entretiens menés auprès de 14 intervenantes francophones du secteur de la VFF. Ces entretiens permettent de commencer à prendre la mesure de leur rôle dans le développement des services en français dans le secteur de laVFF. Les données portent sur les modes d'action des intervenantes ainsi que les savoirs ou apprentissages qu'elles tirent de leurs efforts afin de les transmettre à d'autres et ainsi encourager la réflexion sur les enjeux liés au développement des services en français en Ontario.

Mots clés : développement de services en français, intervenantes, modes d'action, savoirs et apprentissages, violence faite aux femmes, Ontario français, Ontario

\section{Abstract}

The article examines the role played by practitioners involved in developing French language services (FLS) in the area of violence against women in Ontario. Since the 1990s, the sector has been more open to servicing Francophone women and FLS services have grown in important ways. However, few studies have looked at the role played by practitioners in this development. This first analysis, based on the result of interviews with 14 practitioners, helps understand their role in the development of FLS. It discusses strategies used by practitioners as well as lessons learned from their experience. Ultimately, these lessons should be relevant to those involved in the development of FLS and provide a basis for discussing issues related to such development.

Key words: action-based knowledge, development of French services, French Ontario, learning, strategies, practitioners, violence against women

\section{Introduction}

Depuis les années 1990, le secteur de la violence faite aux femmes (VFF) a connu en Ontario des avancées importantes au chapitre 
"Loin d'être le produit d'une action purement volontaire de la part des pouvoirs publics, il nous semble important de voir comment l'action des intervenantes peut constituer un facteur clé en vue du développement des services en français dans le secteur de la $V F F$ » des services offerts dans leur langue aux femmes francophones ${ }^{1}$. Ils se sont multipliés, comme le soulignent de façon régulière des organismes communautaires, entre autres, Action ontarienne contre la violence faite aux femmes (AOcVF), le principal porteparole en violence faite aux femmes pour l'Ontario français ${ }^{2}$. Comment expliquer de tels progrès? Le présent article porte sur les modes d'action des intervenantes francophones ainsi que sur les savoirs ou apprentissages qu'engendrent leurs efforts dans la mise sur pied de services en français en Ontario. Ces questions nous paraissent importantes en vue de contribuer aux travaux sur les facteurs conditionnant le développement des services en français en Ontario (Cardinal, et collab., 2005). Loin d'être le produit d'une action purement volontaire de la part des pouvoirs publics, il nous semble important de voir comment l'action des intervenantes peut constituer un facteur clé en vue du développement des services en français dans le secteur de laVFF. Nous partons donc de l'hypothèse selon laquelle les intervenantes francophones jouent un rôle dans le développement des services en français dans le secteur de laVFF.

L'étude faisant l'objet de cet article s'inspire des recherches effectuées dans le domaine de la gouvernance (Burlone, et collab., 2008; Richard et Rieu, 2008; Paquet, 2009; Forgues, 2015). Par l'expression "mode d'action", ces auteurs entendent d'habitude les règles, les procédures et les rapports, souvent hiérarchiques, qui président à l'action des individus et groupes au sein de structures bureaucratiques, par exemple, celle de l'État (Burlone, et collab., 2008). De plus, des modes d'action correspondent à des formes de rationalité relationnelle, comme des rapports de confiance ou de solidarité et à des activités de délibération. Selon Paquet (2009) et Forgues (2015), ces formes de rationalité non hiérarchiques sont plus fréquentes au sein des groupes et des réseaux d'action communautaire. Elles ont un caractère plus informel, mais l'action des individus et groupes qui repose sur la confiance et la solidarité plutôt que sur la hiérarchie n'est pas sans effet. Elle peut aussi avoir des conséquences pratiques sur le développement des services au public ou des politiques, et plus particulièrement sur 
le développement des services en français dans le secteur de la VFF, comme nous tenterons de le montrer ci-dessous.

L'expression « savoirs ou apprentissages » utilisée dans cet article réfêre à ce que les intervenantes retirent de leur engagement. Ces acquis permettent de mieux jauger les conséquences de leurs actions sur les pratiques du milieu et de revenir sur les enjeux associés aux modes d'action relationnels, en opposition à hiérarchiques, pour le développement des services en français dans le secteur de laVFF.

L'article comprend trois parties. Dans un premier temps, nous situerons notre propos dans le contexte des travaux de recherche réalisés sur les services en français dans le secteur de la VFF en Ontario français. Dans un deuxième temps, nous définirons notre cadre d'analyse et notre stratégie de recherche. Dans un troisième temps, nous proposerons une analyse de nos résultats de recherche. Notre étude se veut exploratoire.

\section{La recherche sur la violence faite aux femmes en Ontario français}

C'est dans les années 1980-1990 que voit le jour un premier corpus de travaux sur les femmes et les groupes de femmes en Ontario français (Coulombe, 1985; Coderre et Hamalian, 1992; Garceau, 1995; Lacelle, 1996; Juteau, 1999). Ce foisonnement des écrits sur et par les Franco-Ontariennes met fin à l'absence de prise en compte des réalités des femmes francophones en milieu minoritaire dans les travaux des féministes canadiennes et québécoises (Cardinal, 1997). La plupart de ces recherches s'inscrivent dans une analyse de la société issue du mouvement des femmes et nourrie par lui selon une perspective féministe.

À ses débuts, la recherche féministe en milieu francophone minoritaire a pour objectif de préciser la spécificité des FrancoOntariennes (Juteau, 1999). L'utilisation du concept de double infériorité, à la fois en tant que femme et en tant que francophone vivant en milieu minoritaire, constitue un apport important des 
«...la transformation radicale tant souhaitée au sein du mouvement des femmes de l'Ontario français a pris le chemin d'une réforme en profondeur du système juridique et le développement de politiques publiques destinées à répondre aux besoins spécifiques des femmes francophones." chercheurs féministes francophones au débat canadien (Cardinal et Cox, 2007). La référence à cette double infériorité connote les rapports de domination qui caractérisent les rapports sociaux de sexes et les rapports sociaux ethniques au sein desquels les femmes francophones de l'Ontario construisent leur identité. Toutefois, la perspective a été lentement délaissée, malgré sa force toujours importante d'explication, pour être remplacée par un deuxième corpus de travaux plus juridiques qui vise à faire reconnaitre les droits des femmes francophones à l'égalité, à la sécurité, à la liberté, à l'intégrité et à la dignité (Côté et Bouchard,1996). En effet, dans la foulée des débats sur la mise en œuvre des droits des femmes stipulés dans la Charte canadienne des droits et libertés de 1982, la transformation radicale tant souhaitée au sein du mouvement des femmes de l'Ontario français a pris le chemin d'une réforme en profondeur du système juridique et le développement de politiques publiques destinées à répondre aux besoins spécifiques des femmes francophones. Par la suite, l'avènement de l'analyse intersectionnelle a contribué à favoriser la prise en compte de la diversité des besoins des femmes francophones (Bunjun, et collab., 2006). Les féministes de l'Ontario français ont alors davantage pris conscience des diverses formes d'oppression vécues par les femmes francophones, en raison de leur appartenance à un groupe « racisé » ou encore à cause de leur orientation sexuelle, en plus de l'oppression vécue comme femme. Les travaux montrent toutefois que les femmes francophones en Ontario ont de la difficulté à faire reconnaitre leur situation de discrimination qui perdure (Cardinal et Cox, 2007).

La recherche féministe francophone a aussi porté une attention particulière au secteur de la VFF, notamment au militantisme des féministes francophones et au rôle clé de groupes comme Action ontarienne contre la violence faite aux femmes (AOcVF) auquel sont liées les participantes à la présente recherche (Bégin 1992; Penwill, et collab., 1997; Brunet, 1998; Brunet et Garceau, 2004; Cardinal, et collab., 2005; Gérome, 2000; Garceau, Savard et Richard, 2012; Garceau et Sirois, 2014). L'histoire d'AOcVF est centrale au développement des services en français enVFF en Ontario français ${ }^{3}$. Fondé en 1988 dans le but de vaincre l'oppression vécue par les femmes, ce regroupement féministe et francophone 
réunit plusieurs organismes (AOcVF, 2014). Son mandat est celui de travailler à la prévention de la violence, à la formation continue des intervenantes et des directions, au démarchage en vue de mettre en place des services en français, à l'analyse des enjeux et à la réalisation de matériel éducatif et de sensibilisation en français, selon une analyse féministe de la situation sociale et communautaire des femmes. Les organismes membres d'AOcVF sont des centres d'aide et de lutte contre les agressions à caractère sexuel (CALACS), des maisons d'hébergement pour les femmes et leurs enfants victimes de violence conjugale ou familiale et des programmes en matière de violence faite aux femmes et aux enfants qui offrent des services en français à travers l'Ontario.

À ses débuts, $\mathrm{AOcVF}$ réunissait les intervenantes des CALACS francophones deux fois l'an. Les maisons d'hébergement francophones et les intervenantes francophones à l'intérieur d'organismes unilingues ou soi-disant "bilingues », choquées du peu de reconnaissance de leurs besoins et de ceux des femmes desservies, se sont jointes aux rencontres des intervenantes des CALACS au milieu des années 1990. Réunies depuis lors, les intervenantes des secteurs de la violence conjugale et de l'agression à caractère sexuel ont pu proposer un discours collectif faisant la promotion des services en français, entre autres, services directs, formation, programmes spécifiques et outils. Si l'étude de l'action des groupes de femmes est bien engagée (Garceau, Savard et Richard, 2012; Coderre, 2014), celle du rôle des intervenantes dans le développement des services en français n'a pas encore fait l'objet d'une attention particulière. Pourtant, ces personnes nous paraissent jouer un rôle important pour la compréhension du développement des services en français. Étant au cœur de l'action, elles sont souvent les mieux placées pour identifier les contraintes au développement des services en français et pour proposer les moyens de les surmonter. L'étude de leurs modes d'action, des savoirs ou des apprentissages des intervenantes travaillant au développement des services en français dans le secteur de laVFF peut recéler des enseignements pertinents pour la compréhension des enjeux liés de façon plus large au développement de services en français. 
"...les chercheuses

féministes ont souvent été mobilisées afin de réaliser des études de besoins, colliger des données et évaluer des projets, ce qui explique que ces travaux représentent aujourd'hui une grande portion de la recherche sur les femmes en Ontario français..."

"...nous constatons que malgré la multiplication des recherches, les intervenantes n'ont toujours pas fait l'objet d'étude, pas plus que leurs modes d'action ou savoirs, d'où l'importance que nous accordons à une telle thématique."
En partie, on peut expliquer cette absence de recherche sur le rôle des intervenantes par les besoins du moment. Dans le secteur de laVFF, la recherche féministe francophone de type communautaire est de loin la plus importante. Cette recherche s'est constituée en réponse à la demande du milieu francophone qui avait un besoin criant de données et de matériaux probants en vue de légitimer ses demandes de services en français auprès des gouvernements (Cardinal, et collab., 2005). Ainsi, les chercheuses féministes ont souvent été mobilisées afin de réaliser des études de besoins, colliger des données et évaluer des projets, ce qui explique que ces travaux représentent aujourd'hui une grande portion de la recherche sur les femmes en Ontario français (Brunet, 1998, 2001; Brunet et Garceau, 2006; Bouchard, Cholette, Younes, 2006; Brunet, Sherwood et Garceau, 2009; Brunet, et collab., 2012). Ils ont permis de comprendre l'ampleur régionale de laVFF en Ontario français et les situations particulières, les défis et les lacunes dans la prestation des services de même que les pratiques les plus efficaces (Garceau et Charron, 2000; Younes, 2004; Grenon, Kérisit et Magunira, 2008; Penwill, 2008; Hashi, 2009; Coderre et Delorme, 2014).Des travaux plus spécialisés ont porté sur certaines problématiques liées à laVFF, comme la prostitution (Day, 2008) ou les préoccupations des femmes immigrantes (Côté, Kérisit et Côté, 2001; Bassolé, et collab., 2004) et des femmes âgées de plus de 65 ans (Charron, 2009). La recherche a aussi permis de mettre en place une formation unique en français et destinée aux intervenantes en $\mathrm{VFF}^{4}$. Sans faire l'objet d'une recension prétendument exhaustive, tous les travaux publiés depuis les années 1980 montrent que les intervenantes sont toujours au cœur de toutes réalisations. Or, nous constatons que malgré la multiplication des recherches, les intervenantes n'ont toujours pas fait l'objet d'étude, pas plus que leurs modes d'action ou savoirs, d'où l'importance que nous accordons à une telle thématique. 


\section{Des savoirs connectés et ancrés : cadre théorique et stratégie de recherche}

Dans les travaux sur la gouvernance, le traitement de la question des modes de coordination de l'action et celle de la rationalité sous-jacente à l'action des actrices et des acteurs, en particulier dans le cadre de leur activité professionnelle, nous paraît pertinent pour le type d'analyse que nous souhaitons proposer dans le présent article (Avenier et Schmitt, 2007; Burlone, 2008; Burlone et Rouillard, 2011; Paquet, 2009; Cardinal et Plante, 2012; Forgues, 2012; 2015). De façon complémentaire, la recherche féministe postule la valeur épistémologique de l'expérience comme fondement d'un savoir privilégié, souvent invisible ou dévalorisé, pour enrichir, voire transformer, nos connaissances (Harding, 1991; Liamputtong, 2007). De plus, la recherche féministe prône l'engagement social (Ollivier et Tremblay, 2000) qui se traduit souvent par la réhabilitation des savoirs expérientiels comme une dimension essentielle dans le développement des stratégies pour influencer le développement de services pour les femmes (Relais-femmes, 2014).

Comme nous lravons mentionné ci-dessus, si la rationalité étatique prend appui sur des rapports hiérarchiques formels, la rationalité communautaire puise son autorité à d'autres sources. Selon Forgues (2012) et Burlone et Rouillard (2011), ces dernières sont multiples et comprennent, entre autres, " les normes de solidarité et de réciprocité "(Forgues, 2012, p. 118) qui s'établissent entre les acteurs; "la confiance entre les acteurs et les liens personnels » (Forgues, 2012, p. 118) tissés entre eux; les valeurs qu'ils partagent entre eux et les objectifs qu'ils ont en communs; les intérêts qu'ils construisent en commun; dans leur influence et poids politique. Ces sources d'autorité fondent la rationalité de l'action communautaire ou influencent les pratiques communautaires. En d'autres termes, elles inspirent des modes d'action plus relationnels, propices à l'action communautaire. Il convient donc de répertorier les modes d'action des intervenantes francophones dans le secteur de la VFF afin de voir d'une part 
s'ils participent de la même logique relationnelle et comment d'autre part ces modes relationnels constituent des pratiques influençant le développement de services en français. Enfin, nous souhaitons comprendre les savoirs ou les apprentissages que tirent les intervenantes de leurs expériences dans le développement des services en français afin de les consigner et de les propager.

La stratégie de recherche choisie est de type qualitatif. Notre ambition était d'interroger des participantes possédant une certaine expérience ainsi qu'un savoir et des connaissances sur le développement des services en français en matière de VFF ayant eu cours dans les vingt dernières années. Pour cette raison, nous avons recruté en priorité des intervenantes occupant des postes de direction. En plus de ce critère principal dans le choix des participantes, s'ajoutait à notre échantillon celui d'une représentation des différentes régions de l'Ontario français. Les données proviennent donc d'entrevues semi-structurées réalisées auprès de 14 intervenantes du secteur de la VFF de l'Ontario français ${ }^{5}$. Précisons que dix des douze organismes représentés par les participantes ont pour mandat d'offrir des services en français seulement, les deux autres offrant aussi des services en anglais. Tous ces organismes sont membres d'AOcVF. En Ontario, les services à l'intention des femmes victimes de violence sont financés principalement par deux ministères : le ministère des Services sociaux et communautaires (MSSC) pour la violence conjugale et le ministère du Procureur général (MPG) pour la violence à caractère sexuel. Parmi les organismes d'où proviennent les participantes, trois reçoivent du financement pour offrir des services en matière de violence conjugale de la part du MSSC et quatre offrent des services en matière d'agression à caractère sexuel avec un financement du MPG. Les cinq autres services sont financés par les deux ministères et s'adressent aux femmes aux prises avec l'un ou l'autre des deux types de violence, ou avec les deux ${ }^{6}$. Trois des organismes représentés sont situés dans le Nord de l'Ontario, quatre dans le Centre-Sud et les autres dans l'est de la province. Parmi les participantes, trois sont des intervenantes de première ligne auprès des femmes et onze sont ou ont été directrices d'organismes communautaires membres d'AOcVF. 
Les entrevues portaient sur l'expérience des intervenantes francophones œuvrant dans le domaine des services en français en VFF, sur le développement des services en français enVFF et sur les savoirs et les apprentissages acquis dans ce domaine. Les entrevues ont été réalisées de la fin novembre 2013 à la fin janvier 2014. Elles ont été transcrites dans leur intégralité, puis dénominalisées. Par la suite, nous avons utilisé l'analyse de contenu (Bardin, 1977; L'Écuyer, 1988), une méthode scientifique, systématique et objective du traitement du matériel recueilli, pour construire les unités de classification ou les thèmes et sous thèmes à être analysés. Ceux-ci ont été constitués à l'aide du logiciel QDA Miner?

\section{Les résultats}

«Qui sont les

intervenantes

francophones et

pourquoi s'engagentelles en VFF? Quels modes d'action ontelles privilégiés pour le développement des services en français en VFF? Quels apprentissages tirent-elles de leur expérience?"
Les entretiens portaient sur les questions suivantes qui recoupent les intentions générales de la recherche : Qui sont les intervenantes francophones et pourquoi s'engagent-elles enVFF? Quels modes d'action ont-elles privilégiés pour le développement des services en français en VFF? Quels apprentissages tirent-elles de leur expérience? Les réponses apportées ont été regroupées et analysées conformément à ces mêmes paramètres.

\section{Les intervenantes}

La moitié des intervenantes, soit sept, sont originaires de l'Ontario français, quatre viennent du Québec et trois sont nées à l'extérieur du Canada. La plupart ont suivi des études de niveau collégial ou universitaire, et ce, dans un domaine lié aux sciences sociales; certaines ont aussi étudié en communication, en administration ou en sciences infirmières. Six d'entre elles possèdent plus de vingt ans d'expérience dans le secteur de laVFF, deux en comptent entre seize et vingt et six possèdent entre six et dix ans d'expérience dans ledit secteur. Les expériences de travail antérieur à leur entrée dans le secteur de laVFF sont variées, mais presque toutes ont œuvré dans le domaine des services sociaux. Beaucoup ont travaillé avec les enfants au sein d'organismes communautaires ou dans le domaine de l'éducation, d'autres en itinérance, en santé 
"On ne peut dire si le féminisme ou l'engagement envers la cause des femmes a été dans tous les cas un facteur de motivation à l'embauche, mais une fois dans le milieu, les intervenantes se sont prises de passion pour leur travail en VFF..." mentale, en immigration ou en désintoxication. Fait à noter, avant leur entrée en poste, la plupart des intervenantes possédaient déjà une expérience de travail ou de bénévolat dans le secteur de la VFF où elles s'étaient initiées à l'approche féministe.

On ne peut dire si le féminisme ou l'engagement envers la cause des femmes a été dans tous les cas un facteur de motivation à l'embauche, mais une fois dans le milieu, les intervenantes se sont prises de passion pour leur travail en VFF :

"En découvrant le féminisme, puis l'analyse féministe, puis le travail auprès des femmes que j'ai trouvé, j'ai découvert ma passion pour mon secteur. " (Intervenante 12)

"C'est quelque chose qui me passionne depuis toujours parce que moi-même étant femme, et je viens d'un pays où la violence faite aux femmes est quotidienne et comme une norme; et dès que j'ai vu le rôle ou bien la mission d'un centre comme tel, ça m'a tout de suite interpellée. » (Intervenante 5)

Si la cause des femmes victimes de violence motive les intervenantes une fois en poste, le fait d'avoir à défendre la légitimité des services en français suscite chez elles d'autres réactions. Pour celles qui sont originaires de l'Ontario français, la revendication pour le développement de services en français est inhérente à leur travail en VFF. Elles ont appris, souvent à un très jeune âge, à défendre leur langue et leur culture. Pour d'autres, travailler à la défense des services en français est le résultat d'une prise de conscience. En effet, si travailler en français est une priorité, avoir à justifier l'existence de services dans cette langue est déstabilisant pour les arrivantes qui n'ont jamais vécu en milieu minoritaire. Une intervenante qui habitait au Québec avant de travailler en Ontario explique qu'elle n'avait pas conscience de la situation des francophones de cette province :

«Ça fait que ç'a été mon entrée, de réaliser que la cause des francophones puis les services 
en français, il fallait les défendre. Ce n'était pas un acquis, tu sais. [...] Mais c'est ça venir d'une culture majoritaire.T’arrives du Québec, puis tu dis tu vas défendre la cause de la violence faite aux femmes; je n'avais jamais pensé que j'aurais aussi une double cause. " (Intervenante 2)

La question des services en français interpelle aussi les nouvelles collègues venues de l'extérieur du Canada :

"J'ai suivi un peu l'histoire où les FrancoOntariens n'avaient pas droit à l'éducation, les stratégies que les Franco-ontariennes ont dû utiliser à un moment donné pour éduquer les jeunes en français, et tout et tout. Je me suis dit bien, oui, ça m'interpelle [...] Les FrancoOntariens ont droit, ont utilisé des stratégies, et c'était important. » (Intervenante 8)

Par ailleurs, travailler en français est un facteur important pour un grand nombre d'intervenantes tant au moment de l'embauche que par la suite :

«Ce qui m'intéressait beaucoup c'était l'idée de travailler en français parce que toutes mes études avaient été faites en français, toutes mes études postsecondaires aussi.» (Intervenante 12)

Par contre, certaines intervenantes prennent aussi conscience que travailler en français en Ontario n'est pas acquis :

"Je me considère extrêmement, extrêmement chanceuse, extrêmement privilégiée parce que

"...en plus de leurs diplômes, plusieurs apportent avec elles un savoir déjà " connecté » et «ancré » sur le milieu.» j'ai pu me créer un environnement francophone [...] Et que j'ai pu travailler, je dirais, en tout temps, ou presque en tout temps, en français." (Intervenante 6)

Ces quelques éléments du profil des intervenantes permettent de voir qu'en plus de leurs diplômes, plusieurs apportent avec elles un savoir déjà " connecté " et " ancré » sur le milieu. Avoir 
fait du bénévolat dans le milieu de la VFF et comprendre les enjeux entourant le développement et l'offre active de services en français en Ontario sont des atouts importants. Toutefois, les entretiens révèlent aussi que les intervenantes, d'où qu'elles viennent, comprennent assez rapidement que travailler en français dans le secteur de laVFF n'est pas suffisant. Une fois embauchées, elles se transforment en actrices pleinement engagées dans le développement des services en français dans le secteur de laVFF.

\section{Les modes d'action}

Les intervenantes sont vite happées par les conditions dans lesquelles elles travaillent. Elles ont une grande conscience de la double oppression des femmes et des francophones et le ressentent dans leur quotidien. Lorsque l'on resitue ces représentations dans le contexte du développement des services en français en VFF en Ontario, leur engagement et leur conscience aiguë de la double oppression des femmes francophones semblent justifiés tellement le domaine a connu un démarrage lent. Rappelons que depuis les années 1970, la majorité des services à l'intention des femmes victimes de violence a été développée pour la majorité linguistique ${ }^{8}$. En 1986, la promulgation de la Loi sur les services en français a été un facteur de motivation qui a conduit plusieurs ministères à s'interroger sur la façon d'offrir des services aux femmes francophones (Cardinal, 2001). Cependant, le réseau de services aux victimes de violence ayant déjà été bien établi au sein de la majorité anglophone, les répondantes sont unanimes à expliquer que convaincre le gouvernement provincial d'ajouter de nouveaux services pour les femmes francophones a été une tâche ardue. Cela dit, en vingt ans, des services en français dans le secteur de la VFF ont vu le jour; si bien qu'aujourd'hui, neuf centres francophones autonomes d'aide et de lutte contre les agressions à caractère sexuel (CALACS) ont pignon sur rue en Ontario; s'y ajoutent trois nouvelles maisons d'hébergement francophones ${ }^{9}$, des programmes d'appui transitoire et de soutien au logement, des programmes pour les mères et enfants, un institut de formation, des programmes de sensibilisation et un centre de services juridiques pour les femmes francophones. Les intervenantes ont déployé des 
"Face à un

contexte difficile, les intervenantes interviewées soulignent qu'elles se sont donné des stratégies d'action."
«Les avancées des intervenantes dans le développement des services en français en VFF semblent dues en partie à leur capacité de se concerter,...» efforts pour la mise en place de ces services. Comment sont-elles intervenues? Comment ont-elles réussi à surmonter les obstacles ou contraintes au développement des services en français? Face à un contexte difficile, les intervenantes interviewées soulignent qu'elles se sont donné des stratégies d'action. Elles ont cherché entre elles à privilégier la concertation et la délibération, notamment, au sein d'AOcVF. Elles se sont aussi donné des normes d'action fondées sur l'adhésion à certains principes communs. Elles ont cherché à créer des alliances avec d'autres groupes ou avec des fonctionnaires et à se doter d'un pouvoir d'influence grâce à l'élaboration d'outils de travail destinés à former et à informer les décideurs de la situation des femmes francophones de l'Ontario ainsi qu'à les guider dans leur prise de décision. En somme, les intervenantes francophones ont misé sur certains facteurs en plus de consacrer leurs énergies à développer une multitude de stratégies en vue de convaincre le gouvernement ontarien de financer des services en français destinés aux femmes francophones. Afin d'illustrer leur rôle dans le développement des services en français, nous présentons ici quelques exemples des différents modes d'action qu'elles ont adoptés.

Apprendre à se concerter : Les avancées des intervenantes dans le développement des services en français enVFF semblent dues en partie à leur capacité de se concerter, parfois dans le cadre de grands rassemblements ${ }^{10}$, mais le plus souvent sous la forme de rencontres semestrielles organisées par AOcVF et réunissant des intervenantes :

«Les intervenantes qui travaillaient sur le terrain se rencontraient deux fois par année, beau temps, mauvais temps. [...] Et c'étaient tous des moments où on se parlait pendant trois jours. C'est quoi les défis? C'est quoi les problèmes? Comment est-ce qu'on pourrait les régler? Et très souvent ça portait sur la question des services en français. » (Intervenante 7) 
Toutefois, la concertation est exigeante. Elle oblige les intervenantes à harmoniser leurs points de vue et leurs stratégies vers un objectif commun.

"La concertation, le fait quand même de développer des approches initiées et coordonnées, le fait d'établir ensemble des plans stratégiques basés sur des besoins réels de la population et puis des plans d'action provinciaux auxquels on collabore. [...] Et je trouve que ça a été des stratégies qui ont quand même contribué comme femmes francophones à nous faciliter; mais chaque fois, on doit travailler doublement plus que la communauté je dirais anglophone ou la communauté majoritairement anglophone. " (Intervenante 4)

Les rencontres de concertation permettent aussi aux intervenantes d'apprendre à se mobiliser et à développer des stratégies. Ainsi, elles acquièrent un pouvoir d'influence sur les processus décisionnels dans leur secteur :

"J'ai développé au cours des années cette capacité-là d'articuler un peu la réalité, d'amener ce qui se fait sur le terrain puis de le faire valoir au niveau un peu plus politique pour qu'on comprenne l'importance de l'obtention de services en français. Parce que quand une femme a souffert de violence quelconque, c'est sûr qu'elle a besoin d'un service dans sa propre langue.» (Intervenante 6)

Parmi les intervenantes à apprivoiser ce pouvoir d'influence, les femmes immigrantes sont de plus en plus nombreuses. À partir du moment où le gouvernement canadien a reconnu que l'immigration francophone pouvait contribuer à la vitalité des communautés francophones hors Québec, les groupes de femmes ont été parmi les premiers à revoir leurs façons de faire et à intégrer les enjeux des nouvelles arrivantes au sein de leurs activités (Cardinal, 1997) : 
"La solidarité et la collaboration avec les femmes immigrantes ont permis un développement de services en français plus inclusif et plus sensible aux enjeux de ces dernières. »

"La norme qui réunit l'ensemble des intervenantes autour d'une vision commune pour le développement des services en français dans le secteur de la VFF se résume à l'expression " par et pour".
«Moi, je viens d'ailleurs. Nous arrivons ici, ces femmes-là arrivent avec des réalités. Ça nous amène maintenant à commencer à travailler sur ces réalités, cette nouvelle réalité ici en Ontario français. » (Intervenante 5)

La solidarité et la collaboration avec les femmes immigrantes ont permis un développement de services en français plus inclusif et plus sensible aux enjeux de ces dernières. Ce faisant, la stratégie a contribué à accroître les pouvoirs et la légitimité des intervenantes francophones dans le secteur de laVFF :

«Et quand je suis arrivée, aussitôt j'étais comme impliquée comme bénévole au niveau des organismes qui aident les francophones ici dans la région, parce que je suis d'abord francophone. Et je m'identifiais à cette communauté-là bien entendu.» (Intervenante 5)

Se donner des normes : En plus de se concerter et d'apprendre à intervenir de façon stratégique, les intervenantes adoptent des normes communes pour guider leurs actions. La norme qui réunit l'ensemble des intervenantes autour d'une vision commune pour le développement des services en français dans le secteur de la VFF se résume à l'expression «par et pour ». Nous insistons pour parler d'une norme au lieu d'une approche ou d'un modèle, car les entretiens révèlent que ce n'est pas tant les moyens qui comptent dans le discours du "par et pour » que l'intention ou son effet mobilisateur. Les intervenantes soulignent que les services en français doivent être conçus par les femmes francophones et pour les femmes francophones. Une intervenante explique comment son groupe a insisté auprès du gouvernement ontarien pour obtenir de tels services :

«Les femmes se sont carrément opposées à l'idée d'encore une fois investir seulement des argents pour des services en français à l'intérieur d'un organisme anglophone. Elles ont clairement identifié que ce n'était pas une formule qui 
fonctionnait, que même s'il y avait l'intention d'offrir des services en français pendant une courte période de temps qu'il n'y avait aucune garantie de la continuité et la qualité des services parce que ça dépendait trop de la philosophie de la personne qui était là sur place. Donc, les femmes ont réclamé la mise sur pied d'un centre francophone qui serait mandaté uniquement pour des services en français, pour assurer que l'offre des services en français se fasse. Donc vraiment c'est l'idée du "par et pour" les francophones. » (Intervenante 12)

Les intervenantes reconnaissent que cette référence au principe du " par et pour " tire sa source d'une double tradition. Dans un premier temps, la montée du mouvement féministe en milieu majoritaire dans les années 1970 a contribué à publiciser une norme semblable selon laquelle les femmes devraient s'organiser entre elles et se doter de services gérés par et pour elles. Ainsi, dans les secteurs de la santé et de la VFF, les groupes féministes ont revendiqué, entre autres, la création de milieux de femmes valorisant les femmes, le respect des femmes et de la culture des femmes et l'abolition du patriarcat, et ce, en éduquant et en informant les femmes, en créant un mouvement collectif et en soutenant des interventions qui visent la reprise du pouvoir et la mise en question des rôles traditionnels. Ce désir de créer des milieux de femmes s'inscrit dans l'approche féministe radicale telle que décrite par Toupin (1998). Plus particulièrement dans le secteur de laVFF, il était acquis que les services devaient être conçus et gérés par et pour les femmes. Ainsi, en Ontario français vers la fin des années 1990, quand les femmes francophones engagées dans le secteur de la VFF se sont levées pour exiger des services gérés dans leur langue par et pour les femmes francophones, elles puisaient dans une tradition féministe qui a été reconnue pour la majorité linguistique (Brunet, 2011; Brunet et Garceau, 2004). Dans un deuxième temps, cette tradition du "par et pour " n'est pas exclusive au secteur de la VFF. Elle s'est aussi étendue dans le domaine de l'éducation en français. Depuis les années 1960, 
la norme du "par et pour " guide l'action des francophones qui partout au pays réclament non seulement le droit à une éducation dans leur langue, mais également le droit à la gestion scolaire, un principe que la Cour suprême a même reconnu en 1990 dans le cadre de l'arrêt Mahé11.

Pour les intervenantes francophones engagées dans le secteur de laVFF, le principe du "par et pour » est donc bien balisé et guide leurs actions. De plus, sur le plan administratif, les intervenantes constatent que lorsqu'elles gèrent leurs propres services en français, elles réussissent à en obtenir un meilleur financement :

«Les autres qui sont demeurés sous la gouverne d'un conseil d'administration anglophone ont stagné ou au bout d'un an ou deux ans ont simplement renoncé à l'enveloppe des services en français. [...] Il y a des endroits où l'argent est encore là, mais c'est encore la même enveloppe qu'en 1994. Il n'y a pas eu de progrès. Alors quand on a constaté que l'offre, quand c'était sous la gouverne des femmes francophones par et pour les femmes francophones, puis je dis francophiles aussi $[\ldots]$ eux autres ont grandi. » (Intervenante 7)

"Les intervenantes ont aussi compris qu'elles ont besoin d'alliées. Elles sont conscientes que ces dernières se font rares, mais certaines ouvrent dans les organismes communautaires de la majorité anglophone."
Se faire des alliées et des alliés : Les intervenantes ont aussi compris qu'elles ont besoin d'alliées. Elles sont conscientes que ces dernières se font rares, mais certaines œuvrent dans les organismes communautaires de la majorité anglophone. Certaines répondantes indiquent qu'il a été possible, au fil du temps, d'établir un rapport égalitaire entre AOcVF et des associations à vocation provinciale, dont l'Ontario Association of Interval and Transition Houses, l'Ontario Coalition of Rape Crisis Centers et le Luke's Place d'Oshawa, afin de mener à bien des projets de collaboration. Toutefois, et malgré ces efforts de collaboration au niveau provincial, il a fallu chaque fois négocier les modalités de collaboration entre les groupes francophones et anglophones :

"On a pu développer des projets avec ces groupes-là, mais ça marche mieux quand on fait 
chacun notre bout, puis qu'on se partage par exemple les grandes orientations, quelque chose comme ça, mais qu'on ne s'attend pas à ce que nous autres on arrive juste après qu'ils ont tout développé le projet puis nous autres, on traduit, tu sais? " (Intervenante 7)

Les intervenantes sont aussi par moments en mesure de se solidariser avec les femmes autochtones :

«Je reconnais dans la communauté autochtone aussi une alliée qui est naturelle pour nous. Parce qu'autant que j'entends les messages qu'ils donnent de la mise sur pied et la gestion des programmes par et pour les personnes autochtones, pour être capable de bien combler leurs besoins puis pas de se faire imposer par la culture blanche les services qui ne vont pas répondre à leurs besoins, c'est le même genre de discours aussi que nous, on véhicule [...] Ça fait que de plus en plus quand on est assis autour de la table, j'essaie d'être très sensible à leurs revendications; puis j'essaie de les appuyer activement aussi parce que souvent leurs revendications ressemblent aux nôtres. " (Intervenante 12)

Ces appuis sont importants, car ils contribuent à légitimer les revendications des intervenantes francophones et à les normaliser en plus de donner accès à un réseau de solidarité à l'extérieur du milieu francophone. Cependant, les intervenantes expliquent qu'il faut aussi travailler de façon étroite avec les fonctionnaires de certains ministères pour les convaincre. Ces derniers jouent un rôle clé, car ils peuvent exercer de la pression sur leurs collègues afin pour voir au financement des services en français, en particulier dans le secteur de laVFF :

"Au niveau politique, on a eu, et on a encore

à l'occasion, des partenaires très impliqués, très

à l'affut de la cause, des services en français 
de la violence faite aux femmes [...] Puis ces gens-là sont souvent pris dans une... Tu sais, le gouvernement c'est quand même, la politique c'est quand même une boîte assez rigide, si on peut mettre ça de même. Puis ils ont des gros jobs, puis ils ne comprennent pas tout le temps les portfolios qu'ils portent. Mais on a eu à l'occasion des gens qui comprennent très bien, qui étaient à l'écoute puis qui nous ont aidées à porter nos causes aux bons endroits, aux bons moments pour faire avancer les choses. " (Intervenante 1)

Les intervenantes se heurtent toutefois à la lenteur des processus lorsqu'elles interviennent sur le plan politique et bureaucratique. Cette lenteur est souvent tributaire des décisions politiques affectant le développement des services en français :

«Quand tu rentres dans l'appareil, ah, mon Dieu, tu sais que si tu pars pour revendiquer quelque chose là, t'en as pour cinq ans. Moi ça, ça me tue quelque part parce que pendant ce temps-là, tu sais que le besoin est là, puis que les femmes attendent, puis les enfants attendent, puis il faut que tu ailles débattre, même si tu sais comment les roues tournent lentement. Ca, c'est ma grande frustration en tout cas.» (Intervenante 2)

Comme nous pouvons le constater, lorsque les intervenantes doivent composer avec la rationalité bureaucratique, cette dernière n'est pas toujours perçue de façon favorable, malgré les alliances. Les milieux gouvernementaux sont souvent des endroits mystérieux difficiles à comprendre et ils génèrent leur lot de frustration pour des femmes d'action qui tentent d'aider d'autres femmes vivant dans des situations d'urgence. Les intervenantes apprennent toutefois à faire de la politique au sein de structures dans lesquelles il est souvent difficile de manœuvrer en y apportant d'autres principes comme ceux de la concertation, de la collaboration, de la solidarité et de la délibération. 
"Elles sont proactives et, de leur propre

initiative, elles soumettent des plans d'action non sollicités aux fonctionnaires et aux ministres. ”
Se donner des outils : En plus des alliances stratégiques avec les fonctionnaires de différents ministères, l'originalité de la démarche des intervenantes tient aussi au fait qu'elles cherchent à anticiper les décisions. Elles sont proactives et, de leur propre initiative, elles soumettent des plans d'action non sollicités aux fonctionnaires et aux ministres. Ainsi, tous les cinq ans environ, et ce depuis 1996, $\mathrm{AOcVF}$ et ses groupes membres au sein desquels se retrouvent les intervenantes, préparent un plan d'action (ou plan de travail comme l'explique une intervenante) qui établit les objectifs qu'ils se fixent en matière de développement de nouveaux services et en ce qui a trait aux services offerts, à la formation, à la prévention et à la sensibilisation :

«Une autre [stratégie] a été de créer des plans de travail qui ont été partagés très ouvertement avec les personnes clés dans les différents ministères. Il s'agissait d'articuler le plan de travail, le plus en détail possible, mais sans non plus que ça devienne un document de 200 pages. D'y aller aussi en priorisant et de soumettre ça régulièrement au gouvernement en disant bien voici sur quoi on va travailler dans les dix prochaines années. Et c'est sûr qu'on ne va jamais l'obtenir à 100 pour cent, mais on en a quand même obtenu de gros, gros morceaux. » (Intervenante 6)

Ces plans d'action constituent des documents fouillés et documentés (Garceau et Sirois, 2014). Pour les appuyer dans ce travail, les intervenantes s'allient aux chercheuses universitaires qui les aident à alimenter leur discours et à préparer des dossiers solides :

"Je pense que les exemples comme des liens tissés avec des universités, des chercheuses, ça nous a aussi porté un grand bien parce qu'on le sait très bien que sans l'appui de recherches, de cet aspect-là qui est plus théorique, scientifique, etc., c'est beaucoup plus difficile de faire valoir le vécu. Mais quand on a ces recherches-là, on est 
beaucoup plus, comment dire, ancré et appuyé.» (Intervenante 6)

«Quand on est articulé dans comment on voit le problème, comment ce problème-là a un impact les gens, les organismes, les populations; et on amène ça vers les instances gouvernementales. Ça aide au niveau du développement des politiques.» (Intervenante 8)

De fait, les intervenantes comprennent le pouvoir des données probantes afin de se donner une plus grande crédibilité auprès des instances gouvernementales. Elles doivent argumenter, présenter des faits et des données solides afin d'alimenter les discussions et les décisions sur le développement des services en français enVFF :

"C'est ça, puis eux autres ont fait toute la compilation, l'analyse, les tendances, qu'est-ce qui ressortait? [...] [Le rapport] m'a tellement facilité la tâche d'aller frapper aux portes puis faire une présentation. Et j'ai dit $\mathrm{OK}$, là je vais vous parler de ce que signifient les besoins juridiques pour les femmes francophones en Ontario. Ça fait que ça émanait de ça, et de ça. Les portes se sont ouvertes. Deux ans plus tard, on a trois bailleurs de fonds qui financent une petite partie du Centre de services juridiques, mais c'est un début. » (Intervenante 10)

La démarche semble conférer de l'autorité et de la légitimité aux intervenantes, car certaines fonctionnaires utilisent maintenant ces divers travaux :

«Une fois, une fonctionnaire a dit : il faut que j'aille argumenter telle affaire au comité inter machin. Dis-moi ce que je peux leur donner comme argument. Ils s'attendent à ça de nous autres. Qu'on les feed quelque part sur nos réalités, nos enjeux, les défis. " (Intervenante 2) 
Ce qui semble convaincre les décideuses et décideurs de contribuer au développement des services en français en VFF c'est l'historique de succès des initiatives mises en place, qu'elles aient été régionales comme la mise en œuvre des CALACS et des nouvelles maisons d'hébergement francophones, ou encore provinciales par l'entremise d'AOcVF :

«Puis maintenant Action ontarienne, avec l'Institut de formation, est en train [de développer] et a développé justement des outils extraordinaires pour travailler avec les jeunes, pour travailler avec les femmes sous toutes sortes de formes ludiques, de documents, de former nos intervenantes aussi. Ça fait que ça, ça a été une grande force aussi pour la communauté francophone en violence.» (Intervenante 2)

Les quelques exemples cités permettent de voir que les intervenantes interviewées, une fois embauchées, s'inspirent de modes d'action fondés sur le rejet de la hiérarchie au profit d'une logique plus relationnelle de fonctionnement. Ce type de pratique leur confere une certaine influence sur le développement des services en français dans le secteur de laVFF. Leurs propos permettent aussi de saisir l'ampleur de leur apport au développement des services en français. Elles apparaissent comme des actrices clés dans leur secteur grâce à leur capacité à favoriser la collaboration, la concertation, la formation et l'information, à prôner une vision commune des services en français, et grâce aussi à leur sens de la stratégie. Elles opèrent selon des modes d'action communautaire qui ont une certaine efficacité. Elles reconnaissent d'ailleurs que leur façon de faire constitue une bonne stratégie :

«Regarde les changements, le nombre de services en français juste en violence faite aux femmes là, dans les dernières 20 années, qu'on a obtenus.» (Intervenante 2) 


\section{Quelques apprentissages}

Les données recueillies à partir des entretiens laissent entrevoir le rôle clé que les intervenantes francophones jouent dans le développement des services en français dans le secteur de laVFF. La spécificité de ce rôle est directement liée à des modes d'action qui puisent leur rationalité dans des pratiques de collaboration, tel que le suggère la littérature. Nous avons aussi voulu dégager des savoirs ou apprentissages évoqués par les intervenantes et qui concernent la représentation de ces modes d'action, leur raison d'être et ce qu'elles en retirent pour la postérité.

Selon Cardinal et Forgues (2015), la question des savoirs et des apprentissages a pris une importance stratégique au sein de la francophonie depuis la fin des années 1990 et cela «n'est pas sans lien avec le contexte social, politique et économique qui s'est complexifié dans les sociétés contemporaines incluant le Canada " (p. 373). Entre autres, les gouvernements fédéral et provinciaux ont multiplié les liens avec les groupes francophones, comme en témoignent les multiples ententes qui guident leurs interventions dans nombre de secteurs, que ce soit ceux de l'éducation, de la justice ou de la santé (Cardinal, et collab., 2008; Forgues, 2010). Cette complexité croissante invite à mobiliser de façon stratégique

"...la notion

de savoirs ou

d'apprentissage porte

sur la réflexivité ou

le retour critique que

les intervenantes

effectuent sur leur

action dans le cadre

de leur travail; ancrés

dans leur expérience

$d u$ développement de

services en français, ces

savoirs sont riches en

enseignements. " les savoirs ou les apprentissages des actrices et des acteurs afin d'élaborer des actions qui profiteront de l'éclairage apporté par ces derniers. Comme le suggère Levin dans Cardinal et collab. (2008, p. 10), « [1] a mobilisation du savoir consiste à fournir les bons renseignements aux bonnes personnes dans le bon format, au bon moment, afin d'influer sur le processus décisionnel ».

Telle qu'évoquée dans notre étude, la notion de savoirs ou d'apprentissage porte sur la réflexivité ou le retour critique que les intervenantes effectuent sur leur action dans le cadre de leur travail; ancrés dans leur expérience du développement de services en français, ces savoirs sont riches en enseignements. Les leçons tirées obligent notamment à prendre conscience de la lourdeur de la tâche. Qu'il s'agisse de collaborer avec les bailleurs de fonds ou avec les groupes de la majorité, les intervenantes interviewées décrivent leurs relations avec l'extérieur en termes révélateurs de 
tous les efforts à déployer pour influencer le développement de services en français au sein de leur secteur d'intervention :

«Qu'il faut toujours se battre. On est vu vraiment quasiment comme des citoyennes de deuxième classe. " (Intervenante 2)

En dépit de la Loi sur les services en français, cette image de soi comme citoyennes de deuxième ordre renvoie à la discrimination historique que vivent les francophones de l'Ontario. Cette dernière se veut systémique tellement elle est ancrée dans les pratiques administratives du gouvernement provincial dont la langue de fonctionnement est l'anglais. De plus, comme en témoigne chaque rapport du Commissaire aux services en français de l'Ontario, ce gouvernement n'a pas toujours le réflexe d'intégrer les services en français dès le moment de la formulation des politiques ou des programmes ${ }^{12}$. Les intervenantes en sont bien conscientes. Pour elles, même le fait d'être bilingues peut devenir un problème et venir annuler le droit des femmes à un service en français :

"Pour les intervenantes, ce n'est pas toujours évident pour elles d'aller réclamer des services en français parce qu'elles sont parfaitement bilingues, parce que c'est plus facile d'aller demander un service en anglais puis de ne pas te faire écœurer. Puis je pense que ça, de développer, ça fait partie, je pense, des éléments que t'as besoin si tu veux travailler dans les services en français, de comprendre c'est quoi l'oppression, de comprendre c'est quoi l'oppression intériorisée, et de pouvoir faire ton propre cheminement par rapport à ça. » (Intervenante 7)

"...les intervenantes confirment qu'il faut développer toutes sortes de stratégies pour faire bouger les décideuses et décideurs. ”
En effet, les intervenantes confirment qu'il faut développer toutes sortes de stratégies pour faire bouger les décideuses et décideurs. Selon les répondantes, ces personnes ne veulent pas toujours comprendre le bien-fondé des services en français, incluant ceux se rapportant à la VFF : 
«On a fait des crises [...] je me suis rendue avec la directrice puis on s'était entendues à l'avance. Elle c'était le bon cop. Oui, elle c'était le bon cop, moi j'étais le bad cop puis on a fait une crise, littéralement. »(Intervenante 6)

Ces " crises " dont il est question dans le cadre des entretiens servent essentiellement à rappeler les besoins des femmes francophones en procédant à des mises en scène dramatiques, comme si ce n'était que la seule façon d'obtenir l'attention des fonctionnaires. Les intervenantes doivent toujours déployer beaucoup d'énergie à convaincre leurs consœurs anglophones au sein de leur secteur communautaire :

«On a encore à expliquer ça à nos collègues anglophones qui, malgré 25 ans d'explications, ont encore de la difficulté à comprendre ce concept-là.» (Intervenante 1)

De fait, pour plusieurs intervenantes, cette incompréhension a parfois des relents de francophobie :

"Quand la personne qui coordonne ce groupe-là me dit : "Mais tu sais, tu es femme immigrante alors..." [...] Pour que tu aies des alliés dans le groupe, il faut amener plutôt le côté immigration et non le côté francophone parce que le côté francophone il y aura personne qui va te soutenir.» (Intervenante 8)

"On entreprend un projet, tout est planifié, toutes les étapes ont été décidées. Mais qu'estce qu'on fait en français? Souvent, tu vois le monde lever les yeux puis dire : "Oh, elle est fatigante, elle! Encore la question des services en français!" " (Intervenante 12)

Les relations avec les bailleurs de fonds et la communauté majoritaire anglophone peuvent donc être lourdes de tensions et d'incompréhension. Les représentations des intervenantes 
«Devant les contraintes, elles déploient des stratégies et n'ont pas froid aux yeux; mais elles doivent aussi en subir les conséquences personnelles, comme celle d'être perçues comme étant "fatigantes »." sont lourdes de sens. Si ces dernières peuvent se solidariser, on ne sent pas que le même climat de confiance règne entre elles et leurs homologues anglophones et les bailleurs de fonds. Ces quelques leçons tirées du retour qu'elles font sur leur expérience de développement de services en français témoignent aussi à quel point elles sont aguerries et qu'elles comprennent les défis auxquels elles font face. Devant les contraintes, elles déploient des stratégies et n'ont pas froid aux yeux; mais elles doivent aussi en subir les conséquences personnelles, comme celle d'être perçues comme étant "fatigantes». En somme, les intervenantes jouent un rôle clé dans le développement des services en français et ce dernier sollicite tout leur être.

\section{Conclusion}

Cette étude sur le rôle des intervenantes francophones dans le développement des services en français dans le secteur de laVFF a permis de voir l'ampleur de leur action et de leur mobilisation. Bien qu'exploratoire, elle montre que les intervenantes se représentent comme des actrices pleinement engagées au sein de leur secteur. Une fois embauchées, elles ne font pas qu'administrer un service. Elles apprennent à devenir des stratèges. Les modes d'action qu'elles ont adoptés, les savoirs qu'elles ont acquis et les apprentissages qu'elles tirent de leur engagement dans le secteur de laVFF sont ancrés dans des rapports de solidarité et ont donné lieu à la création de nouveaux liens de confiance qu'elles ont tissés entre elles. L'effet de ces façons de faire sur le développement des services en français montre qu'elles ont acquis un certain pouvoir d'influence. Ainsi, les données présentées sur les modes d'action et les savoirs ou apprentissages des intervenantes permettent de commencer à tracer un portrait des modalités de l'action des intervenantes envers les services en français dans le secteur de la VFF en particulier ${ }^{13}$. Nous allons poursuivre ce travail d'analyse afin d'approfondir ces résultats et continuer de les documenter.

Cette étude révèle aussi en amont que la tradition de recherche féministe et communautaire en milieu francophone a contribué 
"Cette étude révèle aussi en amont que la tradition de recherche féministe et communautaire en milieu francophone a contribué à "former " toute une génération de femmes engagées envers le développement des services en français. » à "former " toute une génération de femmes engagées envers le développement des services en français. En consignant leurs expériences, leurs savoirs ou apprentissages, nous convenons aussi de l'intérêt stratégique à étudier le rôle des intervenantes dans le développement des services en français. Cet intérêt est stratégique, car les savoirs ou les apprentissages que nous consignons pourraient s'avérer utiles à d'autres personnes. Ces savoirs ou apprentissages mériteraient d'être comparés à ceux d'intervenantes ou d'intervenants dans d'autres secteurs, que ce soit celui des services en français dans le domaine de la santé ou de la justice afin d'approfondir la transférabilité potentielle des modes d'action déployés par les intervenantes du secteur de la VFF et de susciter la réflexion sur leur efficacité.

\section{Notes}

1 Nous remercions le Conseil de recherche en sciences humaines du Canada (CRSH) de son appui à la réalisation de la recherche dont cet article fait l'objet.

2 Voici les organismes en place, leur lieu et leur année de fondation : Maison d'amitié (Ottawa) (1976); Maison Interlude House (Hawkesbury) et Habitat Interlude (Kapuskasing), les CALACS (1983); Centre Victoria pour femmes Sudbury, Oasis Centre des femmes, Toronto, le CALACS francophone d'Ottawa, Espace pour elles, Hamilton et Niagara (1993-1995); Centr'Elles (Centre des femmes francophones du Nord-Ouest de l'Ontario) (1998); Maison d'amitié II (Ottawa), Centre Passerelles à Timmins, programme des Travailleuses en appui transitoire et soutien au logement (TATSL) (2006); Centre Novas (région de Prescott-Russell), Carrefour des femmes du SO (London) (2007); Colibri Centre des femmes (comté de Simcoe) (2008); Villa Renouv'Ellement, Timmins (2010); La Maison, maison d'hébergement pour les femmes francophones de Toronto (2013). (Voir le site : http://aocvf.ca/services-aux-femmes/

3 Au Canada, la première maison d'hébergement pour femmes victimes de violence a vu le jour en 1973 à Toronto, et la même année, les premiers Rape Crisis Center ouvraient leurs portes à Toronto et àVancouver. Entre 1973 et 1978, dix maisons d'hébergement ont ouvert leurs portes en Ontario, dont la Maison d'amitié à Ottawa en 1976, la première maison d'hébergement francophone. Au début des années 1990, on en comptait quatre-vingt-quinze (95), dont Maison Interlude House (Hawkesbury) et Habitat Interlude (Kapuskasing), toutes deux ouvertes en 1983 et offrant des services en français.

Le nombre de maisons d'hébergement en Ontario a peu augmenté depuis ce temps-là, c'est donc dire qu'AOcVF a entrepris ses activités au moment où la majeure partie du financement visant l'offre de services aux femmes victimes de violence avait déjà été accordé en Ontario. 
Du côté des CALACS, le développement s'est aussi fait d'abord du côté anglophone, et parmi les quelque 35 centres, on compte aujourd'hui neuf CALACS francophones, s'ajoutant aux six maisons d'hébergement où une femme francophone peut trouver en tout temps des services offerts dans sa langue.

4 À ce sujet, on peut visiter le site http://ressources-violence.org/formation/

5 Au départ, 16 entrevues ont été effectuées. Pour la rédaction de l'article, comme deux entrevues ont été effectuées auprès d'agentes gouvernementales, nous les avons retranchées du corpus d'analyse. La réalisation des entretiens a été effectuée par Tina Desabrais, postdoctorante à l'Alliance de recherche Les savoirs de la gouvernance communautaire, que nous remercions.

6 Nous notons que dans certaines régions, il y a absence de financement de l'un ou de l'autre ministère, et s'il y a un organisme francophone enVFF, qu'importe l'origine de son financement, il pourrait être appelé à offrir aussi des services aux femmes vivant une problématique de violence pour laquelle l'organisme n'est pas financé.

7 Nous remercions Marie-Hélène Eddie, doctorante à l'Université d'Ottawa, pour son aide à entrer les données dans QDMiner.

8 Citons par exemple l'historique se retrouvant sur le site de l'organisme principal regroupant les maisons d'hébergement anglophones de l'Ontario : «By the early 1990s, there were over 95 women's emergency shelters established in Ontario " (http://www.oaith.ca/about-us/history. html, réf. du 8 mars 2015). À cette date, seules trois maisons d'hébergement (sur ces 95) en Ontario étaient en mesure d'accueillir en français et en tout temps des femmes francophones.

$9 \quad$ Voir le site : http://aocvf.ca/services-aux-femmes/ ainsi que la note ii ci-dessus.

10 Mentionnons les rassemblements suivants : Relevons le défi-Colloque sur l'intervention féministe (Sudbury, 1992); Sensibiliser, décider, agir (Ottawa, 1994); Visibles et Partenaires - Colloque sur les pratiques et les recherches féministes en milieu francophone (Sudbury, 1997); États généraux 2004 : développement des services en français en matière de violence contre les femmes (Ottawa, 2004); Forum provincial sur les services en français en matière des programmes contre la violence faite aux femmes: dialogue sur les meilleures pratiques et les partenariats (2008).

11 Voir le site : http://scc-csc.lexum.com/scc-csc/scc-csc/fr/item/580/index.do — L'arrêt Mahé donne raison aux parents francophones de l'Alberta en ce qui a trait à la gestion des conseils scolaires en 1990.

12 Voir le site suivant : http://csfontario.ca/fr/section/publications

13 AOcVF met des ressources à la disposition des intervenantes afin de les appuyer et de leur donner les outils pour qu'elles puissent travailler en français. Le rôle important d'AOcVF dans l'élaboration d'une stratégie provinciale pour le développement des services en français et l'appui aux intervenantes n'a été qu'effleuré dans cet article et sera élaboré davantage dans le rapport subséquent de recherche.

\section{Bibliographie}

ACTION ONTARIENNE CONTRE LA VIOLENCE FAITE AUX FEMMES (2015). Mandat et mission, réf. du 10 octobre 2014, http://aocvf.ca/qui-sommes-nous/mandat-et-mission/ 
AVENIER, Marie-José, et Christophe SCHMITT (dirs.) (2007). La construction de savoirs pour l'action, Paris, L'Harmattan, coll. «Action et Savoir - Série Rencontres », 245 p.

BARDIN, Laurence (1977). L'analyse de contenu, Paris, PUF.

BASSOLÉ, Angèle, et collab. (2004). L'impact du conflit armé sur l'intégration des femmes immigrantes et réfugiées francophones en Ontario : "On dit toujours que la guerre est finie, mais la guerre n'est jamais finie... ", Ottawa, Mouvement ontarien des femmes immigrantes francophones.

BÉGIN, Monique (1992). "Nouvelles alliances, nouvelles coalitions ", dans Marie-Luce Garceau (dire.), Relevons le défi! Actes du colloque sur l'intervention féministe dans le Nord-Est de l'Ontario, Ottawa, Les Presses de l'Université d'Ottawa, p. 273-279.

BOUCHARD, Lyne, Chantal CHOLETTE et Mila YOUNES (2006). «Étude de besoins : services d'hébergement pour les femmes et leurs enfants fuyant une relation violente dans la région de Toronto ", Convergence, Ottawa, rapport présenté à AOcVF.

BRUNET, Lucie (1998). Les services en français en matière de violence faite aux femmes : le défrichage d'un chemin tortueux, Ottawa, Action ontarienne contre la violence faite aux femmes.

BRUNET, Lucie (2001). Évaluation de huit projets-pilotes financés en vertu du Plan stratégique de développement des services en français en matière de violence contre les femmes, Ottawa, Office des affaires francophones.

BRUNET, Lucie, et Marie-Luce GARCEAU (2004). Faire autant avec si peu... Bilan et profil des services en français en matière de violence contre les femmes, Ottawa, Action ontarienne contre la violence faite aux femmes.

BRUNET, Lucie, et Marie-Luce GARCEAU (2006). Étude de besoins sur les services en français pour les femmes, jeunes et enfants francophones du comté de Simcoe dans le domaine de la violence à caractère sexiste, Toronto, ministère du Procureur général de l'Ontario.

BRUNET, Lucie, David SHERWOOD et Marie-Luce GARCEAU (2009). Étude de besoins sur les services en français pour les victimes francophones de Kingston, Toronto, ministère du Procureur général de l'Ontario, [document non publié].

BRUNET, Lucie, et collab. (2012). Portrait des besoins juridiques des femmes francophones de l'Ontario, Toronto, Centre francophone de Toronto et Ottawa, Action ontarienne contre la violence faite aux femmes.

BUNJUN, Bénita, et collab. (2006). Coup d'œil sur les cadres d'analyse féministe intersectionnelle Intersectional feminist frameworks: An emerging vision, Ottawa, Institut canadien de recherches sur les femmes.

BURLONE, Nathalie, et collab. (2008). «Horizontalité et gouvernance décentralisée :les conditions de collaboration dans le contexte de l'action communautaire ", Administration publique du Canada, Vol. 51, No 1, p. 127-142.

BURLONE, Nathalie, et Christian ROUILLARD (dirs.) (2011). L'État et la société civile sous le joug de la gouvernance, Québec, Les Presses de l'Université Laval, 280 p.

CARDINAL, Linda (2001). Chroniques d'une vie politique mouvementée, Ottawa, Le Nordir, 152 p.

CARDINAL, Linda (1997). L'engagement de la pensée. Écrire en milieu minoritaire francophone, Ottawa, Le Nordir, 196 p.

CARDINAL, Linda, et Éric FORGUES (2015). Gouvernance communautaire et innovations au sein de la francophonie néobrunswickoise et ontarienne, Québec, Les Presses de l’Université Laval, 294 p. 
CARDINAL, Linda, et collab. (2005). Les services en français dans le domaine de la justice en Ontario : un état des lieux, Toronto, ministère du Procureur général.

CARDINAL, Linda, et Rachel COX (2007). «Représentation et légitimité des groupes de femmes francophones vivant en milieu minoritaire au Canada », dans Marie-Blanche TAHON (dire.), Les frontalières, Montréal, Éditions du remue-ménage, p. 96-117.

CARDINAL, Linda, Stéphane LANG et Anik SAUVÉ (2008). "Les minorités francophones hors Québec et la gouvernance des langues officielles : portrait et enjeux ", Francophonies d'Amérique, $\mathrm{N}^{\circ} 26$, p. 209-233.

CARDINAL, Linda, Nathalie PLANTE et Anik SAUVÉ (2010). De la théorie à la pratique : les mécanismes d'offre et de demande des services en français dans le domaine de la justice en Ontario : le point de vue des fonctionnaires et des usagères et usagers, volume 2, Ottawa, Chaire de recherche sur la francophonie et les politiques publiques.

CARDINAL, Linda, et Nathalie PLANTE (2012) « Mobilisation des connaissances au sein de la francophonie ", dans Caroline Andrew, Ruth Hubbard et Gilles Paquet (dirs.), Gouvernance communautaire : innovations dans le Canada français hors Québec, Ottawa, Invenire, p. 95-105.

CHARRON, Françoise, Marie-Luce GAR CEAU et Johanne OUIMETTE (2009). La violence faite aux femmes aînées francophones. Une problématique à cerner, des services en français à offrir, Ottawa, Action ontarienne contre la violence faite aux femmes.

CODERRE, Cécile, et Julie DELORME (2014). Une réalité qu'on ne peut ignorer : portrait régional des violences faites aux femmes des comtés de Prescott-Russel et les services communautaires disponibles pour les femmes et les familles, Prescott-Russell, Coalition de Prescott-Russell pour éliminer la violence faite aux femmes.

CODERRE, Cécile, et Arpi HAMALIAN (1992). "Des femmes de la francophonie ", Recherches féministes, Vol. 5, No 2, p. 1-4.

CÔTÉ, André, et Lyne BOUCHARD (1996). Le droit à l'égalité des Franco-Ontariennes, Ottawa, Table féministe francophone de concertation provinciale de l'Ontario.

CÔTÉ, Andrée, Michèle KÉRISIT et Marie-Louise CÔTÉ (2001). Qui prend pays... L'impact du parrainage sur les droits à l'égalité des femmes immigrantes, Ottawa, Table féministe francophone de concertation provinciale de l'Ontario

COULOMBE, Danielle (1985). "Doublement ou triplement minoritaires ", Revue de l'Université d'Ottawa,Vol. 55, № 2, p. 131-136.

DAY, Shelagh (2008). La prostitution, une violation des droits humains des femmes pauvres, Ottawa, Action ontarienne contre la violence faite aux femmes.

FOR GUES, Éric (2010). «La gouvernance des communautés francophones en situation minoritaire et le partenariat avec l'État ", Politique et sociétés, Vol. 29, №1, p. 71-90.

FORGUES, Éric (2012). "Les apprentissages en gouvernance communautaire ", dans Caroline Andrew, Ruth Hubbard et Gilles Paquet (dir.), Gouvernance communautaire : innovations dans le Canada français hors Québec, Ottawa, Invenire, 2012, p. 115-126.

FORGUES, Éric (2015), " La collaboration interorganisationnelle au sein de la gouvernance communautaire en Acadie au Nouveau-Brunswick », dans Linda Cardinal et Éric Forgues (dir.), Gouvernance communautaire et innovations au sein de la francophonie néobrunswickoise et ontarienne, Québec, Les Presses de l'Université Laval, p. 97-122. 
GARCEAU, Marie-Luce (1995). Franco-Ontariennes de 45 à 64 ans : analyse de leurs conditions de vie, thèse de doctorat, Montréal, Université du Québec à Montréal.

GARCEAU, Marie-Luce, et Marc CHARRON (2000). Pour exprimer son histoire en français. Rechercheaction sur les besoins, les services et les modèles de services en français en matière de violence contre les femmes dans le Nord de l'Ontario, Thunder Bay, Centre des femmes francophones du Nord-Ouest de l'Ontario.

GARCEAU, Marie-Luce, Sébastien SAVARD et Stéphane RICHARD (2012). « Une lutte constante pour contrer les violences faites aux femmes et leur assurer des services en français de qualité : entrevue avec Ghislaine Sirois ", Reflets, revue d'intervention sociale et communautaire, Vol. 18, $\mathrm{N}^{\circ}$ 1, p. 22-37.

GARCEAU, Marie-Luce, et Ghislaine SIROIS (2014). Éliminer la violence faite aux femmes en Ontario français : une tâche ardue. États généraux 2014. État des lieux sur les agressions à caractère sexuel et la violence conjugale : services en français et enjeux prioritaires dix ans après les États généraux de 2004, Ottawa, Action ontarienne contre la violence faite aux femmes.

GÉROME, Marie-Hélène (2000). "La Marche mondiale des femmes en l'an 2000 en Ontario français ", Reflets, revue d'intervention sociale et communautaire, Vol. 6, No 1, p. 192-196.

GRENON, Émilie, Michèle KÉRISIT et Françoise MAGUNIRA (2008). L'analyse des enjeux des femmes immigrantes et réfugiées francophones vivant en Ontario séparées de leurs enfants, Toronto, Mouvement Ontarien des Femmes Immigrantes Francophones.

HASHI, Rahma (2009). Adaptabilité des services en français en matière de lutte contre la violence familiale faite aux femmes de la communauté des minorités raciales et ethnoculturelles francophones - MREF, Toronto, Mouvement ontarien des femmes immigrantes francophones.

HARDING, Sandra (1991). Whose Science? Whose Knowledge?, Ithaca (NY), Cornell University Press, $336 \mathrm{p}$.

JUTEAU, Danielle (1999). L'ethnicité et ses frontières, Montréal, Les Presses de l'Université de Montréal, $232 \mathrm{p}$.

LACELLE, Élisabeth J. (1996). «D'une terre en friche à la serre universitaire : un itinéraire en études des femmes ", dans Linda Cardinal, À partir de notre expérience. Femmes de la francophonie ontarienne, Cahiers réseau de recherches féministes, No 4, Montréal, Université du Québec à Montréal, p. 183-200.

L'ÉCUYER, Robert (1988). "L'analyse de contenu : notion et étapes », dans Jean-Pierre Deslauriers, Les méthodes de la recherche qualitative, Québec, Presses de l'Université du Québec, p. 49-65.

LEVIN, Ben (2008). Réflexions sur la mobilisation du savoir, un document de discussion préparé pour le Conseil canadien sur l'apprentissage et le Conseil de recherche en sciences humaines du Canada, Toronto, Institut d'études pédagogiques de l'Ontario.

LIAMPUTTONG, Pranee (2007). Researching the vulnerable, London, Sage Publications, 258 p.

OLLIVIER, Michèle, et Manon TREMBLAY (2000). Questionnements féministes et méthodologie de la recherche, Paris et Montréal, L'Harmattan, $258 \mathrm{p}$.

PAQUET, Gilles (2009). «Public governance : $(\mathrm{G}=>\mathrm{g}) \cap\left(\mathrm{G}_{1}=>\mathrm{G}_{2}\right)$ », Optimum online, La revue de gestion du secteur public, Vol. 39, $\mathrm{N}^{\circ}$ 4, p. 17-34.

PENWILL, Kathryn, et collab. (1997). "Action politique à la mode de chez nous ", Reflets, revue d'intervention sociale et communautaire, Vol. 3, № 2, p. 166-186. 
PENWILL, Kathryn (2008). Comprendre au lieu d'étiqueter. L'intervention auprès des survivantes d'agression à caractère sexuel psychiatrisées, Ottawa, Action ontarienne contre la violence faite aux femmes.

RELAIS-FEMMES (2014). Le modèle de transfert des connaissances de Relais-femmes, réf. du 3 mars 2015, http://www.relais-femmes.qc.ca/images/files/RELAIS\%20FEMME_livret-2014_VFweb.pdf

RICHARD, Sophie, et Thierry RIEU (2008), Une approche historique de la gouvernance pour éclairer la gestion concertée de l'eau en France, publié dans le cadre du IWRA 13th World Water Congress, Montpellier (France) [en ligne]

http://www.iwra.org/congress/2008/resource/authors/abs863_article.pdf (consulté le 6 mars 2015).

TOUPIN, Louise (1998). Les courants de pensée féministe, réf. du 2 mars 2015, http://www.rcentres. qc.ca/public/les_courants_de_pensée_féministe.html.

YOUNES, Mila (2004). Accessibilité des services en français dans la région d'Ottawa pour les femmes francophones et les femmes immigrantes d'expression française victimes de violence conjugale et de violence à caractère sexuel, Ottawa, Comité Réseau. 